\title{
COMP-Angiopoietin-1 Stimulates Synovial Proliferation but Suppresses Osteoclast by Enhancing Angiogenesis and Osteoblast Maturation in Collagen-Induced Arthritis
}

\author{
Yong-Geun Jeong,"\#, Hyun-Ok Kim ${ }^{1, \#, ~ H y e ~ S o n g ~ L i m ~}{ }^{1}$, Young-Sool Hah', \\ Hee Young Cho ${ }^{2}$, Jiahua Yu ${ }^{3}$, Byung-Hyun Park ${ }^{3}$, Gou Young Koh ${ }^{4}$, Sang-Il Lee ${ }^{1}$
}

Department of Internal Medicine and Institute of Health Science, Gyeongsang National University School of Medicine ${ }^{I}$, Clinical Research Institute, Gyeongsang National University Hospital ${ }^{2}$, Jinju, Department of Biochemistry, Medical School and Diabetes Research Center, Chonbuk National University ${ }^{3}$, Jeonju, National Research Laboratory of Vascular Biology and Department of Biological Sciences, Korea Advanced Institute of Science and Technology, Daejeon, Korea

Objective. Angiopoietin-1 (Ang1) is a potent angiogenic factor that can increase synovial angiogenesis and also enhance osteoblast maturation and bone formation. However, its role in rheumatoid arthritis (RA) has not been well documented. Thus, we investigated roles of Ang1 in collagen-induced arthritis (CIA).

Methods. A recombinant adenovirus carrying the gene that encodes either cartilage oligomeric matrix protein (AdCOMP)-Ang1 (a modified form of Ang1) or LacZ (AdLacZ) was injected intravenously into CIA mice. Clinical, radiological, histopathological, and immunofluorescent analyses were performed. Serum levels of receptor activators of nuclear factor $\kappa B$ ligand (RANKL) and osteoprotegerin (OPG) and expression of osteoblast maturation genes were analyzed.

Results. AdCOMP-Ang1-injected mice developed more severe inflammation than the AdLacZ-injected mice. However, there were no significant differences in cartilage damage and bone erosion. More PECAM-1-positive blood vessels were seen in the synovium of the AdCOMP-Ang1-injected mice

\section{Introduction}

Rheumatoid arthritis (RA) is a major cause of joint destruction and permanent deformity (1). Angiogenesis plays a critical role in the ingress of inflammatory cells and the formation of than in those injected with AdLacZ. Interestingly, a lower number of TRAP-positive osteoclasts were observed in AdCOMP-Ang1-injected CIA mice than in the AdLacZ group when comparing sections obtained from joints showing similar synovial proliferation. The serum OPG/RANKL ratio and expression of osteoblast maturation genes, such as runt-related transcription factor 2 , bone sialoprotein, type 1 collagen, osteopontin, and osterix, were significantly upregulated in the AdCOMP-Ang1 group.

Conclusion. COMP-Ang1 facilitates arthritis onset and increases synovial inflammation, but enhances osteoblast maturation, which in turn inhibits osteoclastogenesis by increasing the OPG/RANKL ratio in CIA. Our results suggest that careful investigation is necessary to delineate the possible therapeutic use of COMP-Ang1 as an adjunctive agent, in combination with anti-inflammatory therapies, for the prevention of bone destruction in RA.

Key Words. Rheumatoid arthritis, Collagen-induced arthritis, COMP-Ang1, Osteoclasts, Osteoblasts

aggressive tumor-like fibrovascular granulation tissue called pannus, which eventually leads to extensive joint destruction (1-3). Thus, angiogenesis inhibition is emerging as a potential therapeutic approach for managing RA. Vascular endothelial

\footnotetext{
<Received : December 19, 2011, Revised (1st: January 20, 2012, 2nd: February 6, 2012), Accepted : February 7, 2012> Corresponding to : Sang-II Lee, Department of Internal Medicine and Institute of Health Science, Gyeongsang National University School of Medicine, 90, Chilam-dong, Jinju 660-702, Korea. E-mail: goldgu@gnu.ac.kr ${ }^{\#}$ The first two authors contributed equally to this study. 
growth factor (VEGF) is the most important and actively studied proangiogenic molecule and angiopoietin-1 (Ang1) and Ang2, which interact with tyrosine kinase with immunoglobulin and epidermal growth factor homology domain 2 (Tie2) receptors, are other important growth factors that enhance tumor angiogenesis $(4,5)$. Several studies have demonstrated that levels of Ang1, Ang2, and Tie2 are increased in RA tissues and that blocking Tie2 activation inhibits angiogenesis and arthritis development in collagen-induced arthritis (CIA) (6-8). Although these data suggest that the Ang-Tie2 pathway is a potential therapeutic target in RA, questions about the unique role of Ang1 in in vivo models of RA remain.

Bone destruction is a major cause of progressive disability in RA patients, and osteoclasts and receptor activators of nuclear factor $\kappa \mathrm{B}$ ligand (RANKL), an essential osteoclastogenic factor, have important roles in RA-related bone destruction $(9,10)$. Bone metabolism is also mediated via the interaction between bone-forming osteoblasts and bone-resorbing osteoclasts (11). The RANKL decoy receptor, osteoprotegerin (OPG), which is expressed by mature osteoblasts, represses the differentiation and activation of osteoclasts by inhibiting RANK signaling (12). In the case of RA, ongoing inflammation within arthritic joints results in a decrease in the number of mature osteoblasts and an enhanced osteoclast function (13). Angiogenesis contributes to bone destruction by allowing the infiltration of inflammatory cells and osteoclast precursors and by inducing cytokines and growth factors (14). Conversely, angiogenesis is closely associated with bone formation, and osteoblast-specific Ang1 overexpression results in increased bone formation (15). However, the role of Ang1 in inflammatory bone destruction has not determined whether it increase or decrease bone destruction.

Cartilage oligomeric matrix protein Ang1 (COMP-Ang1) is a recently developed chimeric form of Ang1 that has more potent and stable activity than native Ang1 (16). COMP-Ang1 leads to the formation of larger, more numerous, and highly branched vessels and increases angiogenesis (17). COMP-Ang1 produces anti-inflammatory effects by enhancing pericyte coverage and by inhibiting vessel leakiness (18). Additionally, COMP-Ang1 enhances osteoblast differentiation and bone formation by promoting angiogenesis and bone remodeling (19-21). COMPAng1, therefore, may either promote or inhibit synovial inflammation and may have some favorable effects on the bone itself due to increased numbers of osteoblasts. However, no existing study has examined the role of Ang1 in an in vivo RA model. In the present study, we investigated the effects of COMP-Ang1 using adenoviral gene transfer in CIA mice.

\section{Materials and Methods \\ Preparation of COMP-Ang1 adenovirus}

Adenoviruses expressing COMP-Ang1 (AdCOMP-Ang1) or bacterial LacZ (AdLacZ) were constructed using the pAdEasy vector system (Qbiogene, Carlsbad, CA, USA) as previously described (17).

\section{Animals and CIA experiments}

DBA/1 mice were purchased from Charles River, and AdCOMP-Ang1 was intravenously injected into normal DBA/1 mice $(n=10)$ to check for any unintended arthritic effects of COMP-Ang1 before the main experiments. CIA was induced as previously described (22). Briefly, male DBA/1 mice (7 to 9 weeks old) were immunized with $150 \mu \mathrm{g}$ of bovine CII (Chondrex, Redmond, WA, USA) emulsified with an equal volume of complete Freund's adjuvant (Chondrex). The day of the first immunization was defined as day 0 . The mice were then boosted with an equal amount of bovine type II collagen emulsified in incomplete Freund's adjuvant on day 21. Mice were divided into three groups: 1) PBS+CIA, 2) AdLacZ+CIA, and 3) AdCOMP-Ang1+CIA ( $n=10$ for each group). PBS or $1 \times 10^{9}$ pfu of either AdLacZ or AdCOMPAng1 were intravenously injected into the tail vein on days 22 and 34 because a previous study showed that serum levels of COMP-Ang1 remained elevated for 2 weeks after AdCOMP-Ang1 injection (23). Clinical arthritis scores were evaluated using a scale of 0 to 4 for each paw, for a total score of 16. Hind paw thickness was measured with an electric caliper placed across the ankle joint at the widest point. On day 45, the mice were sacrificed, and sera and joint tissues were harvested from each animal. All of the experimental animals used in this study were maintained under the protocol approved by the Institutional Animal Care and Use Committee at Gyeongsang National University.

\section{Radiological examination}

Plain radiographs of the paws were obtained using a mammographic imager based on a direct detection flat panel array design (Mammomat NovationDR, Siemens Medical Solutions, Erlangen, Germany) using exposure settings of $30 \mathrm{kVp}$ and $90 \mathrm{mAs}$. The degree of joint destruction and bone erosion was scored on a scale from 0 to 5 , where 0 denotes no damage; 1 , minor bone destruction observed in one enlightened spot; 2 , moderate changes, 2 to 4 spots in one area; 3, marked changes, 2 to 4 spots in multiple areas; 4 , severe erosions afflicting the joint; and 5, complete destruction of the joints. 


\section{Histopathological and morphometric analyses}

Fixed joint tissues were decalcified and embedded in paraffin. Sections $(5 \mu \mathrm{m})$ were stained with H\&E, Safranin-O, and a tartrate-resistant acid phosphatase (TRAP) staining kit (Sigma, St. Louis, MO, USA). The joint sections were scored for changes in synovial proliferation, cartilage damage, and bone erosion, all on a scale of 0 to 3 . The number of TRAP-positive multinucleated cells containing three or more nuclei was counted in 10 areas of each ankle and knee (200× magnification). For immunofluorescent analysis, joints were collected, fixed with $10 \%$ buffered formalin, decalcified, and embedded in Tissue-Tek OCT compound (Sakura, Japan), and then $10 \mu \mathrm{m}$ sections were stained with anti-mouse PECAM-1 antibody (Chemicon International, Temecula, CA, USA). Fluorescent signals were visualized, and digital images were obtained with a FV-1000 confocal microscope equipped with argon and helium-neon lasers (Olympus, Japan). Density measurements of PECAM-1 positive blood vessels in the synovium were made on five randomly selected fields $(200 \times)$ for each knee and ankle joint $(n=5-6$ for each group) using photographic analysis of NIS-elements imaging software (Nikon, Japan). The results were expressed as relative area percent of the total area per field.

\section{ELISAs}

The serum samples were prepared on day 45 of CIA. Tumor necrosis factor (TNF)- $\alpha$, interleukin (IL)- $1 \beta$, OPG, and RANKL concentrations were measured using commercially available ELISA kits according to the manufacturer's instructions (R\&D Systems, Minneapolis, MN, USA).

\section{Real-time RT-PCR}

All CIA mice were sacrificed at day 45, and total RNA was prepared from the ankle joints and first-strand cDNA was generated according the manufacturer's instructions using random hexamer primers provided in the first-strand cDNA synthesis kit (Applied Biosystems, Framingham, MA, USA). Specific primers for each gene (Table 1) were designed using Primer
Express software (Applied Biosystems). The real-time RTPCR reaction mixture consisted of $10 \mathrm{ng}$ reverse-transcribed total RNA, $167 \mathrm{nM}$ forward and reverse primers, and $2 \times$ PCR master mixture in a final volume of $10 \mu \mathrm{L}$. The PCR was carried out in 384-well plates using the ABI Prism 7900HT Sequence Detection System (Applied Biosystems). All of the experiments were performed in triplicate.

\section{Statistics}

Values are expressed as the mean \pm SEM. Fisher's exact test was used to analyze the difference in incidence among groups. The Mann-Whitney U test was used to analyze arthritic severity and the radiographic and histological findings. An unpaired Student's t-test and one-way ANOVA were used to analyze the other results. A $\mathrm{p} \leq 0.05$ was considered significant.

\section{Results}

COMP-Ang1 increased synovial inflammation and proliferation but not bone erosion

AdCOMP-Ang1 had no arthritic effect in normal DBA/1 mice (data not shown). AdCOMP-Ang1 and the control AdLacZ were injected into the tail vein of CIA mice on days 22 and 34. Skin on the face and all paws of the CIA mice injected with AdCOMP-Ang1 was distinctly redder than those of mice treated with AdLacZ (Fig. 1A), although both groups showed comparable development of arthritic swelling and cumulative incidence of arthritis (Fig. 1A and B). When the joint swelling and erythematous change were assessed using an arthritis scoring system, along with hind paw thickness, AdCOMP-Ang1-injected mice showed earlier aggravation and more severe arthritis than AdLacZ-injected mice (Fig. 1C and D). Mice were sacrificed at day 45 , and radiological and histopathological findings were evaluated. AdCOMP-Ang1-injected mice showed increased synovial proliferation. Additionally, there were no differences between the two groups' other pathologic findings, such as cartilage damage, bone erosion, and radiological scores (Fig. 2A-C). Taken together, these re-

Table 1. Sequences and accession numbers for the forward and reverse primers used in real-time RT-PCR

\begin{tabular}{lllc}
\hline \multicolumn{1}{c}{ Gene } & \multicolumn{1}{c}{ Forward } & \multicolumn{1}{c}{ Reverse } & Accession No. \\
\hline Runx2 & GCTCACGTCGCTCATCTTG & ACACCGTGTCAGCAAAGC & NM_009820 \\
BSP & TGAAGAGTCACTGCCTCCCT & GTCTTTAAGTACCGGCCACG & NM_008318 \\
Osteopontin & TGGCTATAGGATCTGGGTGC & ATTTGCTTTTCCTGTTTGG & NM_009263 \\
Type 1 collagen & TAGGCCATTGTGTATGCAGC & ACATGTTCAGCTTTGTGGACC & NM_007742 \\
Osterix & GGACTGGAGCCATAGTGAGC & CTCTCCATCTGCCTGACTCC & NM_130458 \\
Type 2 collagen & GCAAGATGAGGGCTTCCATA & CTACGGTGTCAGGGCCAG & NM_031163 \\
Sox-9 & TCCACGAAGGGTCTCTTCTC & AGGAAGCTGGCAGACCAGTA & NM_011448 \\
GAPDH & CGTCCCGTAGACAAAATGGT & TTGATGGCAACAATCTCCAC & NM_008084 \\
\hline
\end{tabular}


A

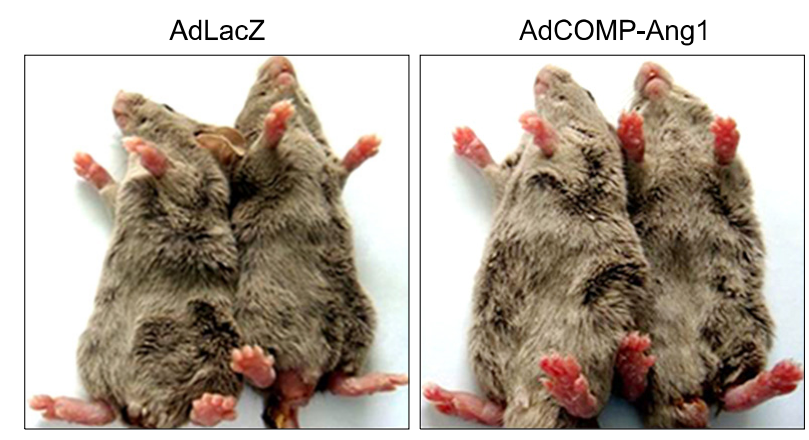

C

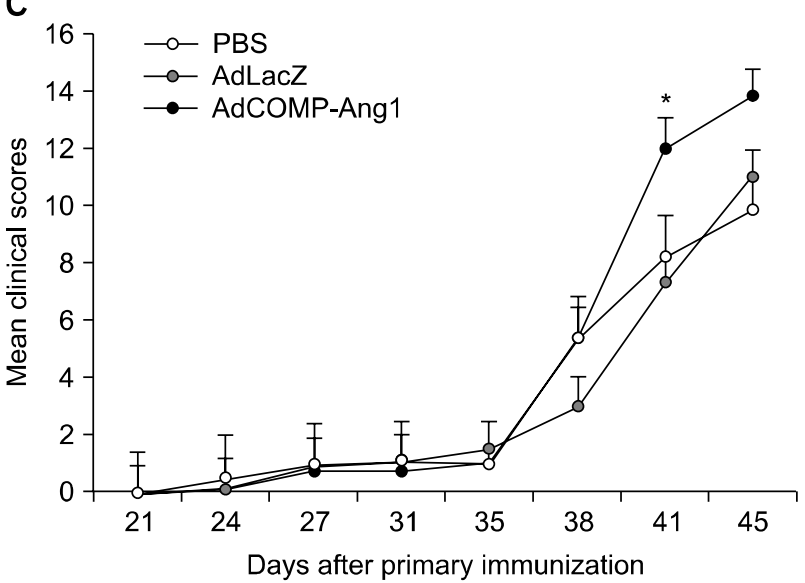

B

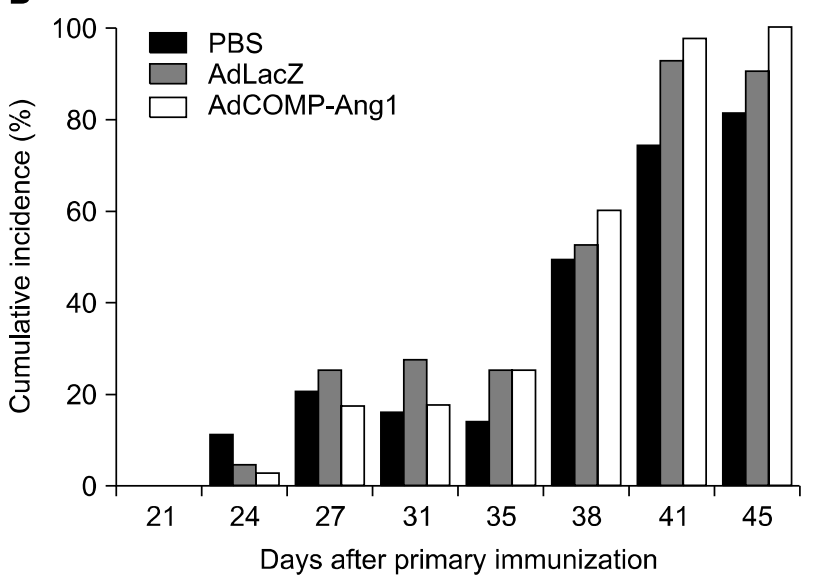

D

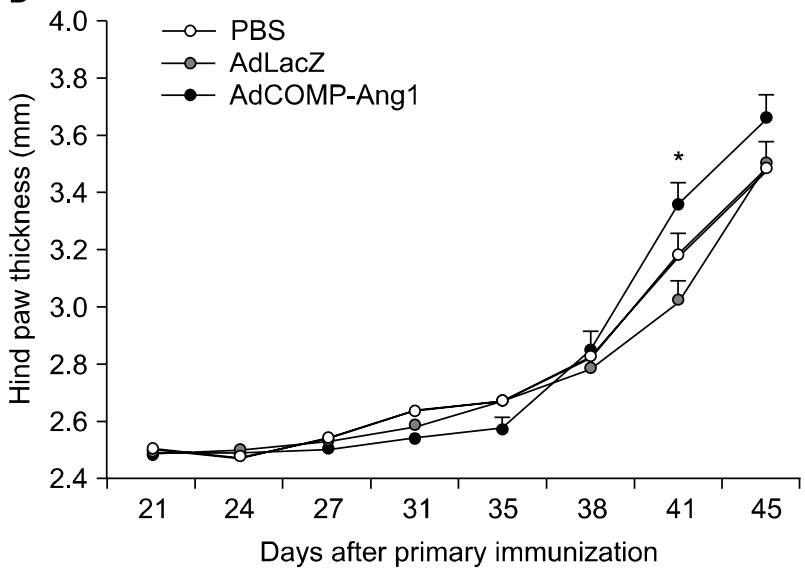

Figure 1. COMP-Ang1 increases erythema and joint swelling. (A) Representative photographs showing the gross features of the hind paws. The mouse injected with AdCOMP-Ang1 shows increased redness (right panel) compared with the Ad-LacZ mouse (left panel), despite showing comparable arthritic development in all paws. (B) The cumulative incidence of arthritis, (C) severity of arthritis, as assessed by a visual arthritis scoring system and (D) hind paw thickness were determined ( $\mathrm{n}=10$ for each group). Values are the mean \pm SEM, *p $<0.05$ vs. AdLacZ.

sults suggest that COMP-Ang1 increases synovial proliferation but not bone destruction in CIA mice.

\section{COMP-Ang1 enhanced synovial vascularity}

AdCOMP-Ang1 increased angiogenesis throughout the synovium of the joints, as shown by an increase in PECAM-1-positive vascular structures (Fig. 2D). Elevated vessel densities in AdCOMP-Ang1-injected mice were further confirmed by morphometric analysis of vessels. A comparison of vascularity found more PECAM-1-positive blood vessels in the synovium of the AdCOMP-Ang1-injected mice than in those injected with AdLacZ (blood vessel density (\%): $4.1 \pm 0.2$ vs. $3.6 \pm 0.2$, $\mathrm{p}<0.05$; Fig. 2E). These findings, taken together with the redder skin color of the paws of AdCOMP-Ang1-injected CIA mice, imply that the increase in arthritic inflammation induced by AdCOMP-Ang1 is attributable to enhanced angiogenesis.

\section{COMP-Ang1 decreased TRAP-positive osteoclasts}

Bone erosion generally occurs in parallel with synovial inflammation and hyperplasia in RA. However, despite the increase in synovial proliferation, there was no notable bone destruction in AdCOMP-Ang1-injected mice. To examine the imbalance between inflammation and bone destruction, we performed pathological assessment on sections obtained from joints showing equivalent degrees of synovial proliferation, particularly grade 3 ( $n=5$ for each group). The results showed that bone erosion was slightly lower in the AdCOMPAng1-injected mice than in those injected with AdLacZ (Fig. 3B). Because osteoclasts are responsible for bone erosion, TRAP staining was performed to determine the number of osteoclasts present in the joints. Interestingly, among joints with equivalent degrees of inflammation, TRAP-positive osteoclasts were reduced in size and number in AdCOMP-Ang1-injected 
A
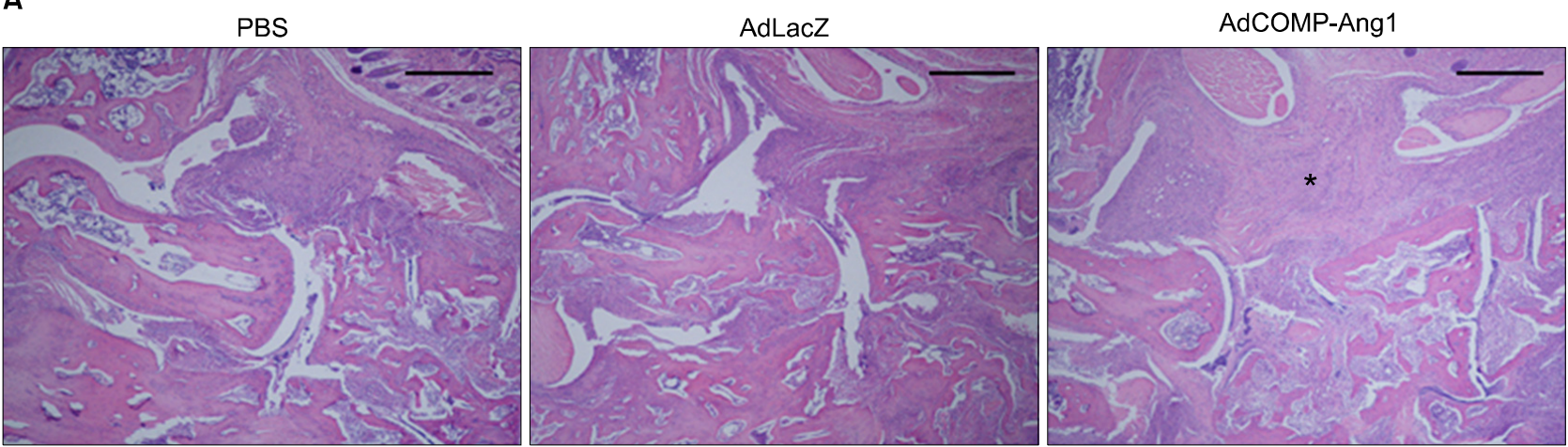

B

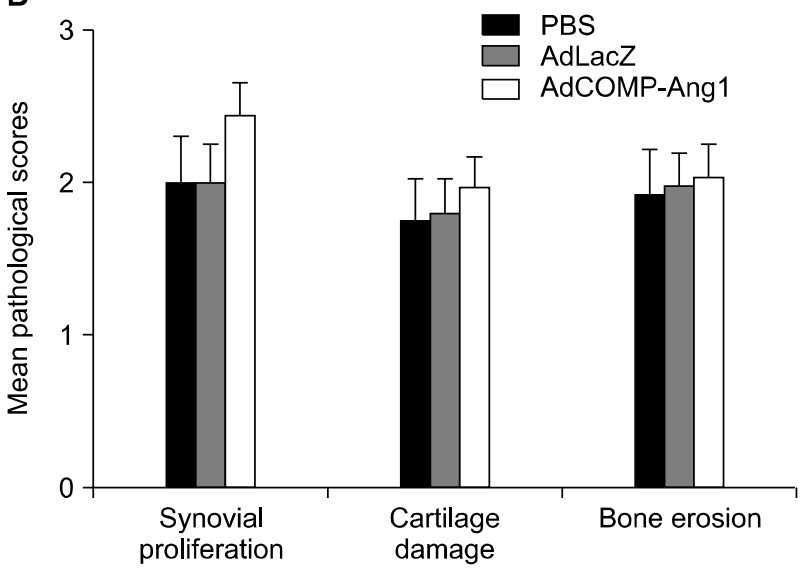

D

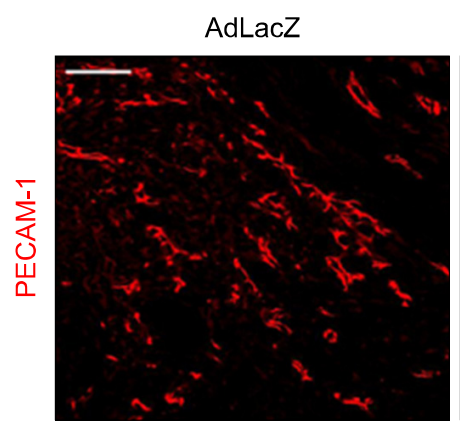

C

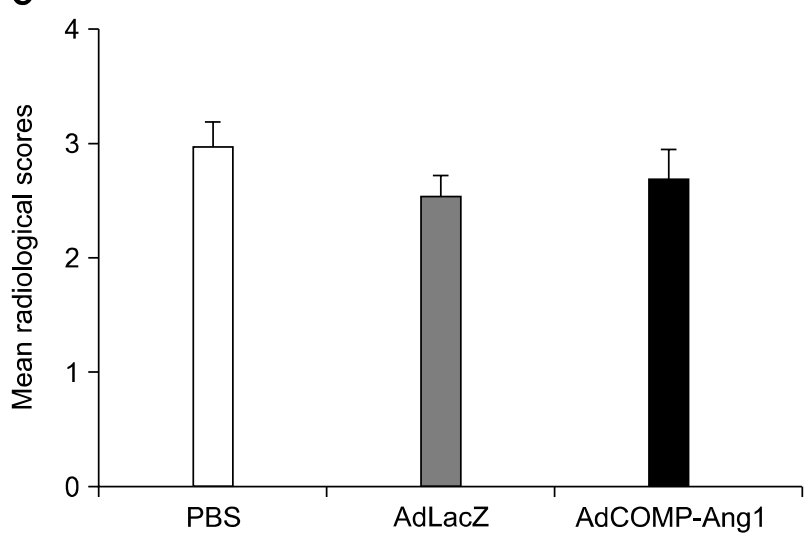

E
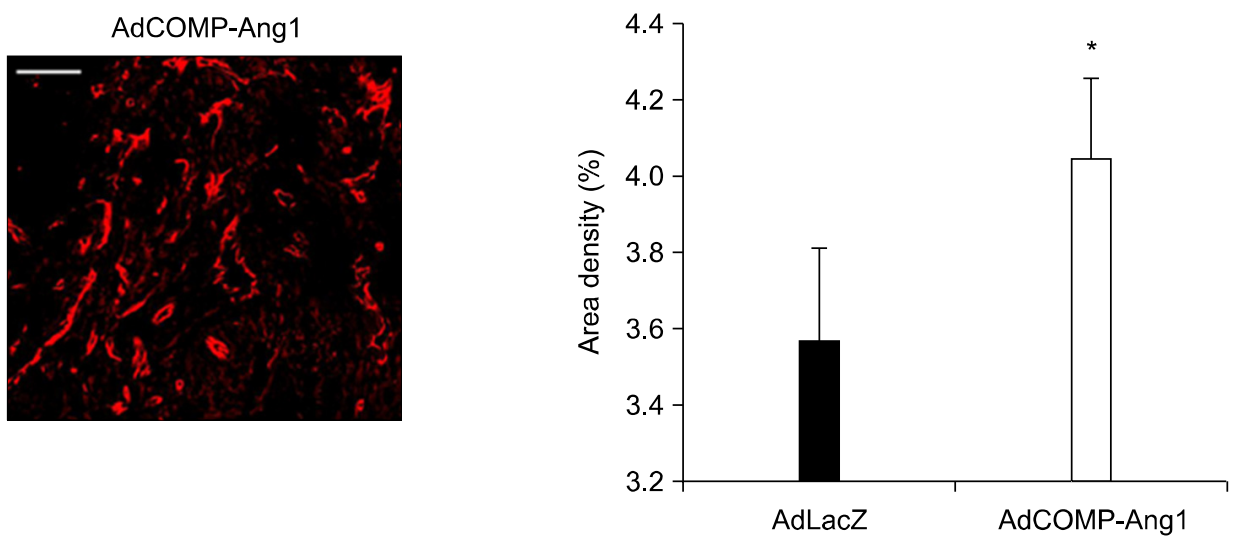

Figure 2. AdCOMP-Ang1-injected mice show increased synovial proliferation and angiogenesis but no bone destruction. (A) Representative sections of the ankle joints stained with H\&E (bars=250 $\mu \mathrm{m}$ ), (B) mean pathological scores, and (C) mean radiological scores ( $\mathrm{n}=10$ for each group). Note the increased synovial proliferation (asterisk) in the joint of an AdCOMP-Ang1-injected mouse compared with PBS- and AdLacZ-injected mice. (D) Images showing PECAM- $1^{+}$blood vessels (bars $=50 \mu \mathrm{m}$ ). (E) Density of blood vessels in the synovium ( $\mathrm{n}=5-6$ for each group). Note the increased angiogenesis in the synovium of an AdCOMP-Ang1-injected mouse compared with an AdLacZ. Values are the mean \pm SEM, ${ }^{*} \mathrm{p}<0.05$ vs. AdLacZ.

mice compared with those in the AdLacZ-injected mice (Fig. 3A). The quantitative analysis indicated that a lower number of TRAP-positive osteoclasts were present in the AdCOMPAng1 group than in the control group (TRAP-positive cells in AdLacZ- vs. AdCOMP- Ang1-injected mice: 221.3 \pm 20.7 vs. $139.3 \pm 13.8, p<0.05$; Fig. 3 C). Collectively, these data demonstrate that COMP-Ang1 increased synovial inflammation and proliferation but decreased osteoclastogenesis in CIA. 
A
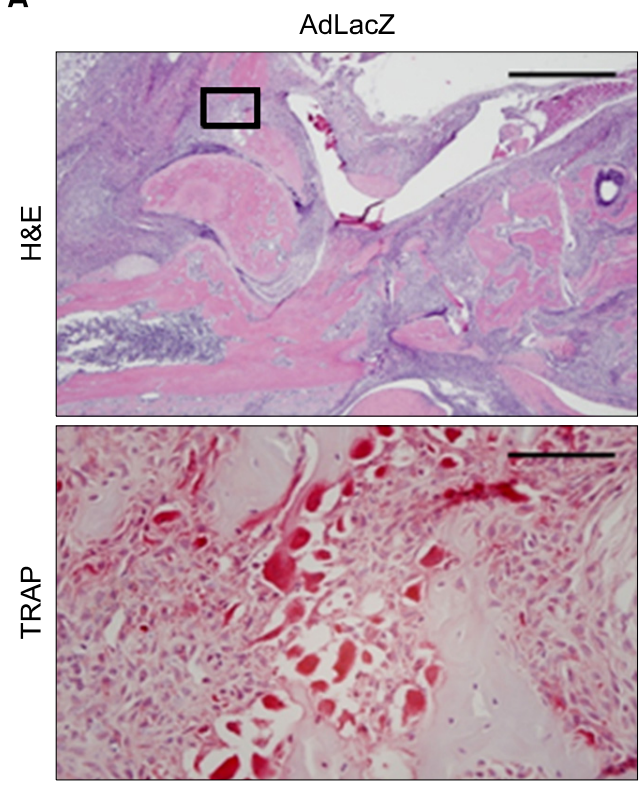

B

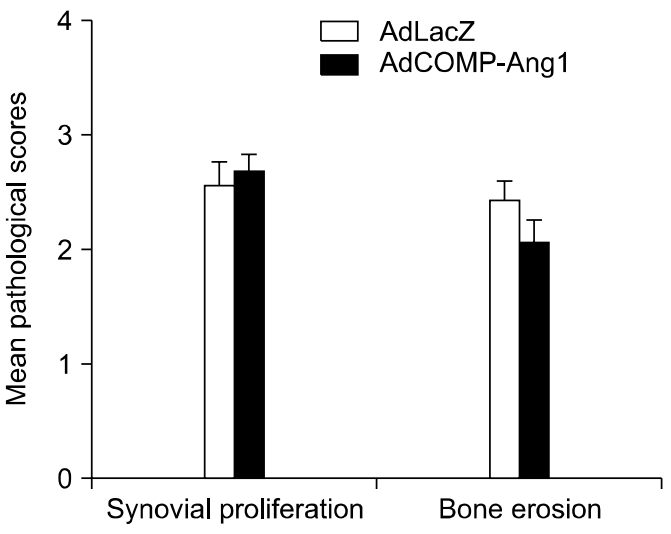

AdCOMP-Ang1

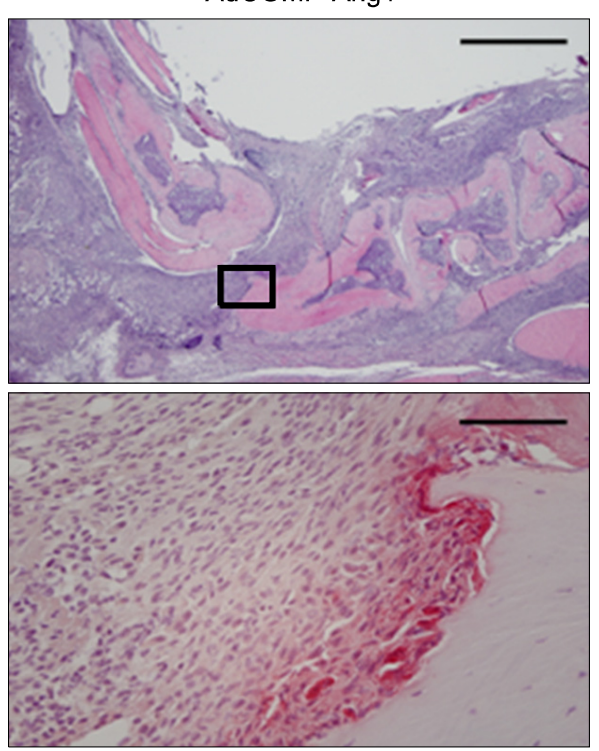

C

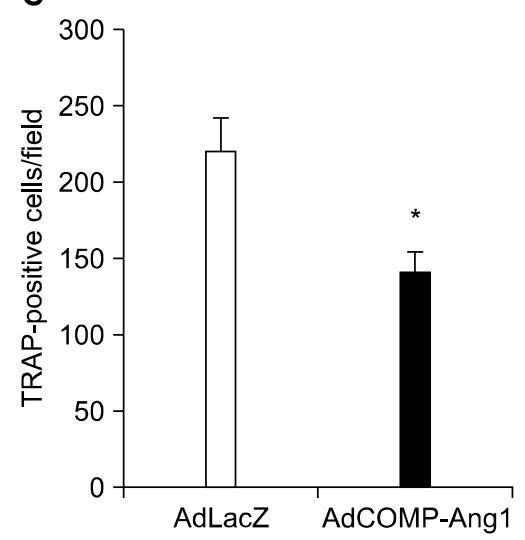

Figure 3. COMP-Ang1 decreases TRAP-positive osteoclasts. (A) Representative sections of the ankle joints stained with H\&E (bars $=500$ $\mu \mathrm{m}$ ) and TRAP (bars $=50 \mu \mathrm{m}$ ) from an AdLacZ- or AdCOMPAng1-injected mouse with grade 3 synovial proliferation. The TRAPstained image (lower panel) corresponds to the boxed area in the H\&E-stained sections (upper panel). (B) Mean pathological scores and (C) total number of TRAP-positive cells in the joints showing grade 3 synovial proliferation from the AdLacZ- or AdCOMP-Ang1-injected groups ( $\mathrm{n}=5$ for each group). Values are the mean \pm SEM, *p $<0.05$.
COMP-Ang1 increased serum OPG/RANKL ratio and osteoblast maturation genes

Serum was collected, and the levels of TNF- $\alpha$ and IL-1 $\beta$ and the OPG/RANKL ratio were measured by ELISA. RNA from the ankle joints was isolated, and the expression levels of several osteoblast maturation genes were examined by real-time RT-PCR. These genes included runt-related transcription factor 2 (Runx2), bone sialoprotein (BSP), type 1 collagen, osteopontin, and osterix. The OPG/RANKL ratio was significantly increased, and osteoblast maturation genes were upregulated in the AdCOMP-Ang1-injected mice (Fig. 4). However, COMP-Ang1 did not affect serum concentrations of TNF- $\alpha$ (AdLacZ vs. AdCOMP-Ang1 mice: $14.5 \pm 1.8$ vs. $16.8 \pm 2.5 \mathrm{pg} / \mathrm{mL}$, value $=$ mean \pm SEM) and IL- $1 \beta$ (288.4 \pm 49.5 vs. $282.5 \pm 58.7)$. We also measured expression levels of chondrogenic genes such as type 2-collagen and SOX-9. There was no significant difference between AdCOMP-Ang1- and AdLacZ-injected mice (Fig. 4). These results indicate that COMP-Ang1 enhances the maturation of osteoblasts and osteogenesis, which in turn inhibits osteoclastogenesis by increasing the OPG/RANKL ratio.

\section{Discussion}

We examined the effects of COMP-Ang1 as an angiogenic and osteoblast-maturating factor on synovial proliferation and bone destruction in a CIA model. We found that COMP-Ang1 increased synovial proliferation but decreased osteoclastogenesis. We also showed that the suppressive effect of COMP-Ang1 on osteoclastogenesis was mediated by the induction of osteoblast maturation and differentiation, followed by an increase in OPG/RANKL ratio. Our results demonstrate the unique role of Ang1 in CIA and suggest the possibility of COMP-Ang1 being used as an adjunctive therapeutic agent to prevent bone destruction in RA.

Inhibition of angiogenesis has been extensively studied for the treatment of a variety of cancers and has begun to emerge 
A

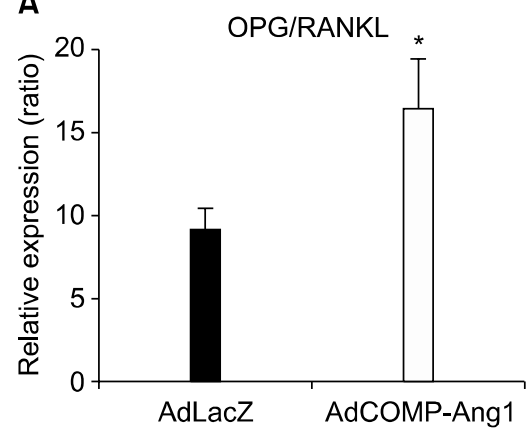

D

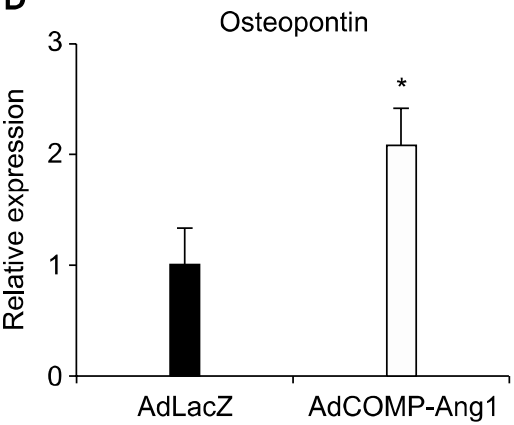

G

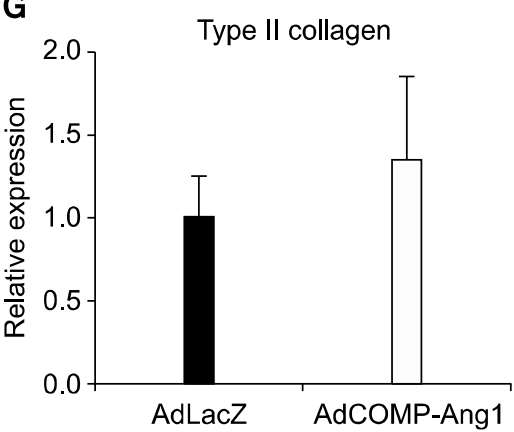

B

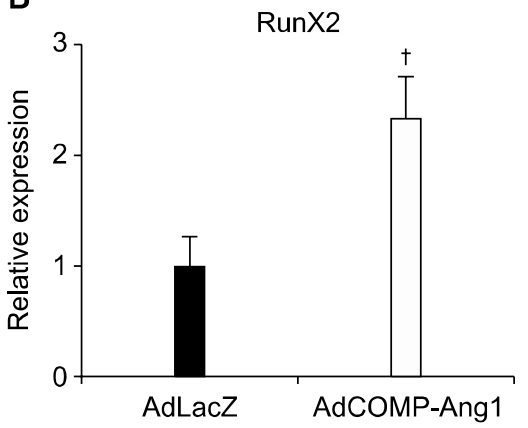

E

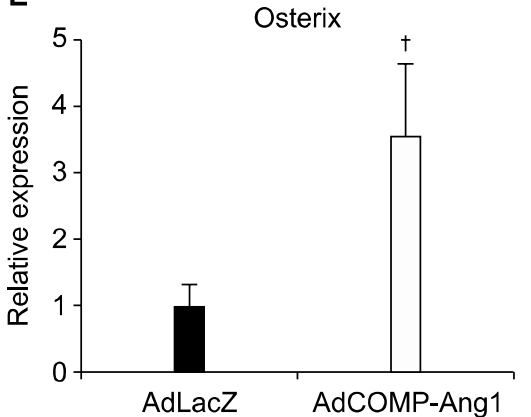

$\mathrm{H}$

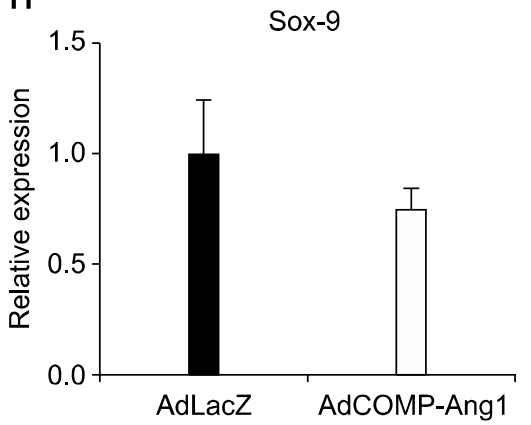

C

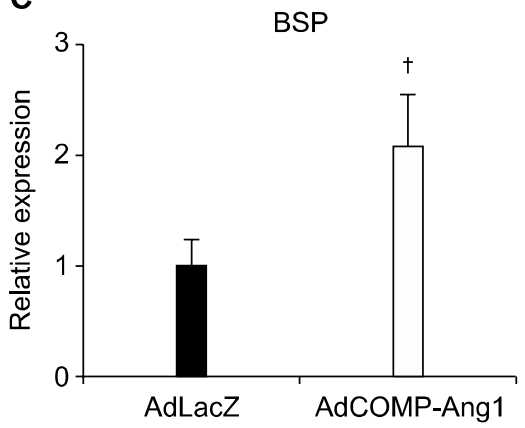

F

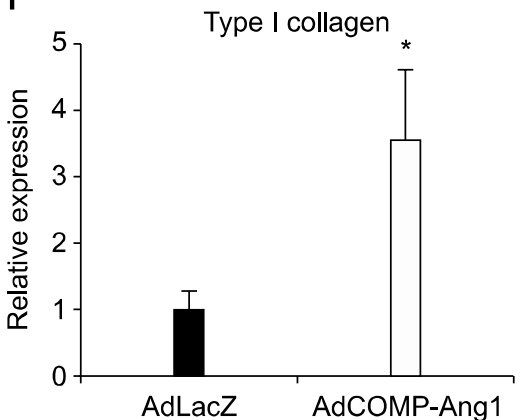

Figure 4. COMP-Ang1 increases the OPG/RANKL ratio and the expression of the osteogenic genes. Ankle joint tissues were obtained from AdLacZ- and AdCOMP-Ang1-injected mice on day 45 ( $\mathrm{n}=10$ for each group). Real-time RT-PCR analysis for osteogenic and chondrogenic genes was performed. Values are the mean \pm SEM, ${ }^{*} \mathrm{p}<0.05,{ }^{\dagger} \mathrm{p}<0.01$ vs. AdLacZ.

as a potential therapeutic approach for managing inflammatory arthritis, particularly RA (24,25). Apart from their activity, much attention has been focused on the side effects, which include hypertension, proteinuria, thromboembolism, bleeding, and gastrointestinal perforations (26). Osteoporosis and impaired fracture healing should be also considered as important side effects of VEGF-inhibitors because its potential as an antirheumatic therapeutic option has become increasingly recognized (25,26). Angiogenesis inhibitors blocking the Ang1-Tie2 pathway may have similar deleterious effects on bone because osteoblast-specific Ang1 increases osteoblast maturation and bone formation (15). Thus, it will be necessary to clearly identify the roles of Ang1 in inflammatory bone and joint diseases such as RA. We found that COMP-Ang1 increases synovial inflammation and proliferation due to enhanced synovial angiogenesis but decreases osteoclastogenesis and bone destruction indirectly via mature osteoblasts. Our results suggest the possibility that osteoporosis and impaired fracture healing may occur as adverse effects of selective inhibition of Ang1.

Some studies have reported stimulating effects of Ang1 on tumor growth, whereas others showed varying degrees of reduced tumor growth $(27,28)$. COMP-Ang1 itself contributes to pericyte coverage and vascular maturation, suggesting that it has an anti-inflammatory propensity (16-18). Thus, Ang1 may either promote or inhibit synovial inflammation and hyperplasia in RA. We found that AdCOMP-Ang1-injected CIA 
mice exhibit an earlier onset of disease and more severe synovial inflammation and proliferation in gross and pathological findings. We also observed increased synovial vascularity and non-suppressed levels of TNF- $\alpha$ and IL-1 in AdCOMPAng1-injected CIA mice. These results are consistent with previous reports showing that Ang1 enhances rheumatoid synovium overgrowth and that Tie2 overexpression causes a psoriasis-like phenotype involving epidermal hyperplasia and inflammatory cell accumulation $(29,30)$. In the context of inflammatory arthritis, angiogenic mediators include proinflammatory cytokines, growth factors, chemokines, extracellular matrix molecules, matrix-degrading enzymes, and cellular adhesion molecules (3). Our results suggest that COMP-Ang1 enhances pathologic angiogenesis and increases synovial inflammation and proliferation when various proinflammatory cytokines are consistently upregulated in CIA.

In addition to synovial hyperplasia, a major clinical manifestation of RA is the progressive destruction of bone, and these two features tend to show a parallel relationship. Angiogenic stimulation also leads to the recruitment of osteoclast precursors and their development into resorptive osteoclasts in the joint (1-3). However, it has been reported that the number of osteoclasts in the bone of Ang1-transgenic mice does not differ from wild-type littermates and that Ang1 increases bone mass by increasing angiogenesis (15). We found no aggravation in bone destruction despite an increase in synovial proliferation and angiogenesis in AdCOMP-Ang1 injected mice. We also performed TRAP staining for osteoclasts in joints with the same clinical score to examine the imbalance between inflammation and bone destruction. Notably, we demonstrated a reduced number of osteoclasts in the AdCOMP-Ang1-injected group, which indicates that Ang1 overexpression may inhibit osteoclastogenesis.

It is well known that the administration of COMP-Ang1 enhances osteoblast differentiation and maturation (19-21). Runx2 is essential for osteoblastic differentiation and positively regulates the expression of BSP, type 1 collagen, osteopontin, and osterix (31). BMP2 is a central morphogenetic regulator of postnatal osteogenesis and increases transactivation activity of Runx2 (32). It has previously been reported that COMP-Ang1 enhances BMP2-induced osteoblast differentiation and bone formation (19). Therefore, COMPAng1 may accelerate osteoblast maturation through BMP2mediated osteogenic gene induction. We found that the expression levels of several osteoblast maturation genes, Runx2, BSP, type 1 collagen, osteopontin, and osterix, were increased in AdCOMP-Ang1-injected CIA mice. The osteoclastogenic potential of immature osteoblasts is mediated by increased
RANKL expression, and mature osteoblasts have relatively low osteoclastogenic activity due to increased OPG expression (33). We also confirmed that the serum OPG/RANKL ratio was increased in AdCOMP-Ang1-injected CIA mice. Taken together, our results suggest that COMP-Ang1 could suppress osteoclastogenesis indirectly via the inhibitory action of the RANKL decoy receptor OPG, which is expressed by mature osteoblasts. However, our study did not show the direct effects of COMP-Ang1 on osteoclasts and these should be investigated further.

\section{Conclusion}

Although existing evidence suggests that COMP-Ang1 can facilitate osteoblast maturation and new bone formation, these effects of COMP-Ang1 on bone have not been previously reported in RA. We showed that COMP-Ang1 enhances the maturation of osteoblasts, which in turn inhibits osteoclastogenesis by increasing the OPG/RANKL ratio in representative animal model of RA. However, we also found that COMPAng1 increase synovial inflammation and proliferation via increased synovial vascularity. Thus, our results suggest that careful investigation is necessary to delineate possible therapeutic use of COMP-Ang1 as an adjunctive agent, in combination with anti-inflammatory therapies, for the prevention of bone destruction in RA.

\section{Acknowledgements}

This work was supported by the Basic Science Research Program through the National Research Foundation of Korea (NRF) and funded by the Ministry of Education, Science and Technology (MEST) [KRF-2008-313-E00293 (SIL)] and a 2008 clinical research grant from Gyeongsang National University Hospital (SIL).

\section{References}

1. Firestein GS. Evolving concepts of rheumatoid arthritis. Nature 2003;423:356-61.

2. Lainer-Carr D, Brahn E. Angiogenesis inhibition as a therapeutic approach for inflammatory synovitis. Nat Clin Pract Rheumatol 2007;3:434-42.

3. Szekanecz Z, Koch AE. Mechanisms of Disease: angiogenesis in inflammatory diseases. Nat Clin Pract Rheumatol 2007;3:635-43.

4. Jones N, Iljin K, Dumont DJ, Alitalo K. Tie receptors: new modulators of angiogenic and lymphangiogenic responses. Nat Rev Mol Cell Biol 2001;2:257-67.

5. Augustin HG, Koh GY, Thurston G, Alitalo K. Control of vascular morphogenesis and homeostasis through the angiopoietin-Tie system. Nat Rev Mol Cell Biol 2009;10: 165-77. 
6. DeBusk LM, Chen Y, Nishishita T, Chen J, Thomas JW, Lin PC. Tie2 receptor tyrosine kinase, a major mediator of tumor necrosis factor alpha-induced angiogenesis in rheumatoid arthritis. Arthritis Rheum 2003;48:2461-71.

7. Gravallese EM, Pettit AR, Lee R, Madore R, Manning C, Tsay A, et al. Angiopoietin-1 is expressed in the synovium of patients with rheumatoid arthritis and is induced by tumour necrosis factor $\alpha$. Ann Rheum Dis 2003;62:100-7.

8. Chen Y, Donnelly E, Kobayashi H, Debusk LM, Lin PC. Gene therapy targeting the Tie2 function ameliorates collagen-induced arthritis and protects against bone destruction. Arthritis Rheum 2005;52:1585-94.

9. Romas E, Gillespie MT, Martin TJ. Involvement of receptor activator of NFkappaB ligand and tumor necrosis factor- $\alpha$ in bone destruction in rheumatoid arthritis. Bone 2002;30:340-6.

10. Takayanagi H. Osteoimmunology and the effects of the immune system on bone. Nat Rev Rheumatol 2009;5:667-76.

11. Choi Y, Arron JR, Townsend MJ. Promising bone-related therapeutic targets for rheumatoid arthritis. Nat Rev Rheumatol 2009;5:543-8.

12. Glass DA 2nd, Bialek P, Ahn JD, Starbuck M, Patel MS, Clevers $\mathrm{H}$, et al. Canonical Wnt signaling in differentiated osteoblasts controls osteoclast differentiation. Dev Cell 2005;8:751-64.

13. Walsh NC, Reinwald S, Manning CA, Condon KW, Iwata $\mathrm{K}$, Burr DB, et al. Osteoblast function is compromised at sites of focal bone erosion in inflammatory arthritis. J Bone Miner Res 2009;24:1572-85.

14. Pap T, Distler O. Linking angiogenesis to bone destruction in arthritis. Arthritis Rheum 2005;52:1346-8.

15. Suzuki T, Miyamoto T, Fujita N, Ninomiya K, Iwasaki $\mathrm{R}$, Toyama $\mathrm{Y}$, et al. Osteoblast-specific Angiopoietin 1 overexpression increases bone mass. Biochem Biophys Res Commun 2007;362:1019-25.

16. Cho CH, Kammerer RA, Lee HJ, Steinmetz MO, Ryu YS, Lee SH, et al. COMP-Ang1: a designed angiopoietin-1 variant with nonleaky angiogenic activity. Proc Natl Acad Sci U S A 2004;101:5547-52.

17. Cho CH, Kim KE, Byun J, Jang HS, Kim DK, Baluk P, et al. Long-term and sustained COMP-Ang1 induces long-lasting vascular enlargement and enhanced blood flow. Circ Res 2005;97:86-94.

18. Thurston G, Suri C, Smith K, McClain J, Sato TN, Yancopoulos GD, et al. Leakage-resistant blood vessels in mice transgenically overexpressing angiopoietin-1. Science 1999;286:2511-4.

19. Jeong BC, Kim HJ, Bae IH, Lee KN, Lee KY, Oh WM, et al. COMP-Ang1, a chimeric form of Angiopoietin 1, enhances BMP2-induced osteoblast differentiation and bone formation. Bone 2010;46:479-86.

20. Park BH, Jang KY, Kim KH, Song KH, Lee SY, Yoon
SJ, et al. COMP-Angiopoietin-1 ameliorates surgery-induced ischemic necrosis of the femoral head in rats. Bone 2009;44:886-92.

21. Park BH, Yoon SJ, Jang KY, Kim MR, Lee HS, Kim $\mathrm{KB}$, et al. COMP-angiopoietin-1 accelerates bone formation during distraction osteogenesis. Bone 2010;46: 1442-8.

22. Hah YS, Lee YR, Jun JS, Lim HS, Kim HO, Jeong YG, et al. A20 suppresses inflammatory responses and bone destruction in human fibroblast-like synoviocytes and in mice with collagen-induced arthritis. Arthritis Rheum 2010;62:2313-21.

23. Lee S, Kim W, Kim DH, Moon SO, Jung YJ, Lee AS, et al. Protective effect of COMP-angiopoietin-1 on cyclosporine-induced renal injury in mice. Nephrol Dial Transplant 2008;23:2784-94.

24. Folkman J. Angiogenesis: an organizing principle for drug discovery? Nat Rev Drug Discov 2007;6:273-86.

25. Carmeliet P. Angiogenesis in life, disease and medicine. Nature 2005;438:932-6.

26. Eskens FA, Verweij J. The clinical toxicity profile of vascular endothelial growth factor (VEGF) and vascular endothelial growth factor receptor (VEGFR) targeting angiogenesis inhibitors; a review. Eur J Cancer 2006;42:3127-39.

27. Hawighorst T, Skobe M, Streit M, Hong YK, Velasco P, Brown LF, et al. Activation of the tie 2 receptor by angiopoietin-1 enhances tumor vessel maturation and impairs squamous cell carcinoma growth. Am J Pathol 2002;160: 1381-92.

28. Machein MR, Knedla A, Knoth R, Wagner S, Neuschl E, Plate KH. Angiopoietin-1 promotes tumor angiogenesis in a rat glioma model. Am J Pathol 2004;165:1557-70.

29. Voskas D, Jones N, Van Slyke P, Sturk C, Chang W, Haninec A, et al. A cyclosporine-sensitive psoriasis-like disease produced in Tie2 transgenic mice. Am J Pathol 2005; 166:843-55.

30. Hashiramoto A, Sakai C, Yoshida K, Tsumiyama K, Miura Y, Shiozawa K, et al. Angiopoietin 1 directly induces destruction of the rheumatoid joint by cooperative, but independent, signaling via ERK/MAPK and phosphatidylinositol 3-kinase/Akt. Arthritis Rheum 2007;56:2170-9.

31. Komori T. Regulation of skeletal development by the Runx family of transcription factors. J Cell Biochem 2005;95:445-53.

32. Bais MV, Wigner N, Young M, Toholka R, Graves DT, Morgan EF, et al. BMP2 is essential for post natal osteogenesis but not for recruitment of osteogenic stem cells. Bone 2009;45:254-66.

33. Thomas GP, Baker SU, Eisman JA, Gardiner EM. Changing RANKL/OPG mRNA expression in differentiating murine primary osteoblasts. J Endocrinol 2001;170:451-60. 